\title{
P01-3-6 Poster session
}

\section{Carbon monoxide reduces ER stress through suppression of Fyn in acute kidney injury}

\author{
Eun-Seon Park, Md Jamal Uddin, Hunjoo Ha \\ Graduate School of Pharmaceutical Sciences, College of Pharmacy, Ewha Womans University, Seoul, Korea
}

Background: Acute kidney injury (AKI), which is defined by a rapid decline of renal function, is common and closely intertwined with chronic kidney disease. However, current treatment for AKI is largely supportive. Since a) endoplasmic reticulum (ER) stress has emerged as a novel mediator of AKI, b) activation of Fyn contributes to ER stress, and c) carbon monoxide inhibits ER stress, the present study investigated the effect of carbon monoxide releasing molecule2 (CORM2) on Fyn-mediated ER stress in AKI.

Methods: Wild type C57BL/6J mice or proximal tubular epithelial (mProx) cells were pretreated with CORM2 or PP2, a Src kinase inhibitor, and administrated with LPS. In addition, cells transfected with control or Fyn siRNA were pretreated with CORM2 and stimulated with LPS. Blood samples were subjected for plasma creatinine and cystatin C, and kidney tissues or cells were used for mRNA and protein analysis.

Results: Pretreatment of CORM2 effectively prevented LPS-induced AKI including decreased glomerular filtration rate, increased oxidative stress and inflammation in mice. CORM2 treatment also effectively inhibited LPS-induced Fyn activation as well as ER stress in AKI mice. Consistently, pretreatment of CORM2 attenuated LPS-induced Fyn activation, ER stress, oxidative stress, and inflammation in mProx cells. In addition, inhibition of Fyn significantly attenuated LPS-induced ER stress both in vivo and in vitro.

Conclusion: These data suggest that CORM2 may become an effective therapeutic agent against AKI through inhibiting Fyn-mediated ER stress.

Keywords: AKI, carbon monoxide, ER stress, Fyn 